\title{
Flexible boundary layer using exchange for embedding theories. II. QM/MM dynamics of the hydrated electron
}

\author{
Zhuofan Shen, ${ }^{1,2}$ Shaoting Peng, ${ }^{1}$ and William J. Glover ${ }^{1,2,3, a)}$ \\ 1) NYU Shanghai, 1555 Century Ave., Shanghai, 200122, China \\ ${ }^{2)}$ NYU-ECNU Center for Computational Chemistry at NYU Shanghai, 3663 Zhongshan Road North, Shanghai, \\ 200062, China \\ ${ }^{3)}$ Department of Chemistry, New York University, New York, NY, 10003, USA
}

The FlexiBLE embedding method introduced in the preceding companion paper [Z. Shen and W. J. Glover, J. Chem. Phys. X, X (2021)] is applied to explore the structure and dynamics of the aqueous solvated electron at an all-electron density functional theory QM/MM level. Compared to a one-electron mixed quantum/classical description, we find the dynamics of the many-electron model of the hydrated electron exhibits enhanced coupling to water $\mathrm{OH}$ stretch modes. Natural Bond Orbital analysis reveals this coupling is due to significant population of water $\mathrm{OH} \sigma *$ orbitals, reaching $20 \%$. Based on this, we develop a minimal frontier orbital picture of the hydrated electron involving a cavity orbital and important coupling to 4-5 coordinating $\mathrm{OH} \sigma^{*}$ orbitals. Implications for the interpretation of the spectroscopy of this interesting species are discussed.

\section{INTRODUCTION}

The hydrated electron, $e_{\mathrm{aq}}^{-}$, an excess electron embedded in liquid water, is the quintessential system exhibiting solvent-supported electronic states, and has been used as an ideal probe of water's solvation dynamics, since the electron itself has no internal degrees of freedom that might muddy a pump-probe spectrum. Nevertheless, a reasonable question to ask is whether the excess electron is simply a spectator in water's solvation dynamics, or whether it strongly perturbs the electronic and molecular structure of the surrounding water to an extent not thermally accessible in the neat liquid.

Much of our understanding of $e_{\mathrm{aq}}^{-}$'s dynamics has come from one-electron mixed quantum classical (MQC) simulations, which consider the quantum mechanics only of the excess electron, while treating the solvent molecules classically. ${ }^{1-10}$ The low computational cost of MQC methods has allowed for converged sampling of the equilibrium properties and dynamics of $e_{\mathrm{aq}}^{-}$, as well as its nonadiabatic dynamics following photoexcitation. ${ }^{2,3,5,8,10}$ Nevertheless, an underlying approximation in all current MQC descriptions of $e_{\mathrm{aq}}^{-}$is that water's electronic structure and thus intramolecular potential energy surface is unchanged from the neat liquid. MQC is thus inherently incapable of addressing the question of whether the excess electron is a spectator, since it is assumed to be.

In addition to the assumption of a neat-liquid water electronic structure, MQC methods rely also on approximate electron-molecule pseudopotentials to describe the interaction of the excess electron with the closed-shell water molecules. While much effort has been devoted to their development, ${ }^{1,4,6-9}$ these pseudopotentials introduce large sources of uncertainty in the accuracy of MQC descriptions of $e_{\mathrm{aq}}^{-}$. Unlike typical atomic pseudopotentials that replace core electrons, the electron-molecule

\footnotetext{
a)Electronic mail: william.glover@nyu.edu
}

pseudopotentials replace valence electrons, which introduces an uncontrolled error. Furthermore, the structural predictions of MQC simulations of $e_{\mathrm{aq}}^{-}$depend quite sensitively on the pseudopotential, $, 8,11,12$ which one of us found recently to arise from a competition of entropic and enthalpic driving forces in the solvation structure of $e_{\mathrm{aq}}^{-} \cdot 13$

A first-principles description of $e_{\mathrm{aq}}^{-}$is thus preferred, and several ab initio molecular dynamics (AIMD) strategies have emerged recently, including Quantum Mechanics/Molecular Mechanics (QM/MM), ${ }^{14,15}$ periodic planewave Density Functional Theory (DFT), ${ }^{16,17}$ periodic second-order Möller-Plesset theory (MP2), ${ }^{18}$ and Path Integral Molecular Dynamics (PIMD) with MachineLearned (ML) potentials. ${ }^{19}$

Given the computational cost of ab initio methods, explorations of the equilibrium dynamics of $e_{\mathrm{aq}}^{-}$have so far been limited, although the initial localization dynamics has received attention. ${ }^{14,16,18,20}$ An exception is the very recent PIMD study of $e_{\text {aq }}^{-}$with ML potentials, which afforded extensive sampling due to the forcefield-like cost of ML potentials. ${ }^{19}$ Of the AIMD methods, QM/MM is particularly efficient, since most of the solvent molecules far from the electron can be treated with a cheap MM forcefield, and only the first few solvent shells around $e_{\mathrm{aq}}^{-}$need to be treated at a QM level. However, a problem arises since water is diffusible, and without a special treatment, the QM and MM water molecules would mix, leading to a breakdown of QM/MM separation. This limits the length of trajectory that can be simulated to a few ps.

The issue of QM/MM mixing by diffusible particles is precisely addressed by our FlexiBLE method, introduced in the previous companion paper. ${ }^{21}$ Thus, to explore the influence of an excess electron on water's electronic structure and dynamics, we performed many-electron FlexiBLE-QM/MM AIMD simulations at the BH\&HLYP/6-31++ $\mathrm{G}^{*}$ level on a model of the condensed-phase $e_{(\mathrm{aq})}^{-}$. We find that the equilibrium dynamics of the electron exhibits enhanced coupling to $\mathrm{OH}$ 
stretch modes compared to an MQC description. By performing Natural Bond Orbital (NBO) analysis, we show that this coupling arises from significant occupation of $\mathrm{OH} \sigma^{*}$ antibonding orbitals, in a similar fashion to that observed in water anion clusters. ${ }^{22}$ This allows us to develop a minimal frontier orbital description of $e_{(\mathrm{aq})}^{-}$, involving a cavity orbital and strong coupling to $4-5 \mathrm{OH} \sigma^{*}$ orbitals. $e_{(\mathrm{aq})}^{-}$can thus be viewed as a strong hydrogen bond acceptor.

The remainder of the paper is as follows. In section II we benchmark DFT for electron-water interactions. In section III we describe the computational details of our condensed-phase model of $e_{(\mathrm{aq})}^{-}$. Results are presented in section IV, where we first apply FlexiBLE to a QM/MM treatment of neat water at the BH\&HLYP /6-31+G* level in section IV A and then to a QM/MM description of $e_{(\mathrm{aq})}^{-}$in section IV B. Dynamics of $e_{(\mathrm{aq})}^{-}$are explored in section IV C, and the NBO analysis is discussed in section IV D. Finally, conclusions are drawn in section V.

\section{BENCHMARKING DFT DESCRIPTIONS OF ELECTRON-WATER INTERACTIONS}

DFT electronic structure has a sufficiently low computational cost that merits its use in AIMD; however, for weakly-bound electronic states one might be concerned about the accuracy of standard functionals. In particular, delocalization error is known to be quite severe for cluster models of the hydrated electron. ${ }^{23,24}$ This motivates a benchmarking of functionals in describing electron-water interactions.

One of us previously developed a method for computing single water-electron interaction energies with varying average relative displacement. ${ }^{9}$ The idea is to include in the electronic Hamiltonian a confining potential of the form:

$$
\hat{V}^{\text {confine }}=\frac{1}{2} k\left(\hat{x}^{8}+\hat{y}^{8}\right)+\frac{1}{2} k_{z} \hat{z}^{8}+C_{z} \hat{z},
$$

where $k$ and $k_{z}$ control the strength of the confinement in the $x, y$ and $z$ directions respectively. In addition, a linear term in $\hat{z}$ is added with strength $C_{z}=-0.015167$ Hartree $\mathrm{Bohr}^{-1}$ that serves to localize the excess electron on one side of the molecule. The $+z$ direction was always chosen to point from the oxygen atom to the electron. The high order of the position operators in Eq. 1 introduces a steep repulsive wall surrounding the water molecule that confines the excess electron. This is needed since a single water molecule alone does not bind an excess electron. A value of $k=1 \times 10^{-7}$ Hartree $\mathrm{Bohr}^{-8}$ confines the excess electron within a few $\AA$ of the water, while leaving the neutral water's electronic structure essentially unperturbed..$^{25} k_{z}$ is then varied between $1 \times 10^{-7}$ and $1 \times 10^{-10}$ Hartree $\mathrm{Bohr}^{-8}$, which has the effect of moving the minimum in the confining potential, and therefore the excess electron's density, to increasing displacement from the water molecule (see dashed red curves in Fig. 2 below).

With the confining potential of Eq. 1, electronic structure calculations were then performed on a water anion. Since the excess electron localizes up to several Ångstom from the water, it is important to augment the standard atom-centered basis functions to describe highly diffuse orbitals. Following our previous work ${ }^{9}$ we achieve this by adding to the aug-cc-pVTZ basis ${ }^{26}$ a cubic grid of $s$ type primitive Gaussian functions with exponent 0.16 Bohr $^{-2}$. The grid contains $7 \times 7 \times 13$ functions with a spacing of 2.5 Bohr. The electron-water interaction energy is defined as:

$$
E^{\text {int }}=E^{\text {anion }}-E^{\text {neut }}-E^{1 e},
$$

where $E^{\text {anion }}$ and $E^{\text {neut }}$ are the total energies of the anionic and neutral systems respectively, and $E^{1 e}$ is the ground-state eigenvalue of a single electron in the confining potential of Eq. 1. We found previously that unrestricted MP2 results agreed well with gold-standard Coupled Cluster Singles Doubles and perturbative Triples $(\operatorname{CCSD}(\mathrm{T}))^{27}$ interaction energies, ${ }^{9}$ so we use MP2 as the reference in this work. Density functional calculations were performed in Q-Chem 5.0, ${ }^{28}$ with the SG1 Exchange-Correlation (XC) integration grid. ${ }^{29}$ Care had to be taken due to our use of a Gaussian Grid basis formed with distributed ghost atoms that each contributed to the integration grid. To avoid a dummy atom getting too close to a water atom and reducing the accuracy of the integration grid, we removed the dummy atom at the origin, and added its $s$ primitive function to the oxygen atom's basis, which was shifted to the origin.

We consider first the BLYP functional, ${ }^{30,31}$ since this was used in the first planewave DFT study of $e_{(\mathrm{aq})}^{-} \cdot{ }^{16}$ Fig. 1 plots the electron-water interaction energy for three different orientations (indicated by the molecular graphics at the top of the figure). Panel (a) shows the interaction energies for an electron on the oxygen side of water. As expected, the interaction energy is positive, corresponding to a repulsion from the partially negative oxygen atom. However, BLYP (dashed blue curves) significantly underestimates the magnitude of repulsion by $\sim 0.2 \mathrm{eV}$ compared to the reference MP2 results (green circles) at high confinement strengths (small $e^{-}$-water distances). Similar behavior is seen in two other orientations: dipole aligned (panel (b)) and $\mathrm{OH}$ bond aligned (panel (c)), which show attractive interactions as expected, but with BLYP overestimating the attraction by $\sim 0.2 \mathrm{eV}$ when the electron is close to the water.

The overestimated attraction between the electron and water at short range in BLYP is suggestive of the delocalization error. Indeed, Johnson and co-workers found significant convexity error in the energy versus fractional charge of a Kevan model of the hydrated electron with BLYP, raising the concern that delocalization error is severe when this functional is applied to solvated electrons. ${ }^{23,24}$ We confirm this is the case in Fig. 2, which plots spin densities for the electron-water system. We in- 


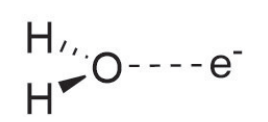

--- BLYP

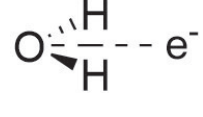

- BH\&HLYP

Interaction Potential
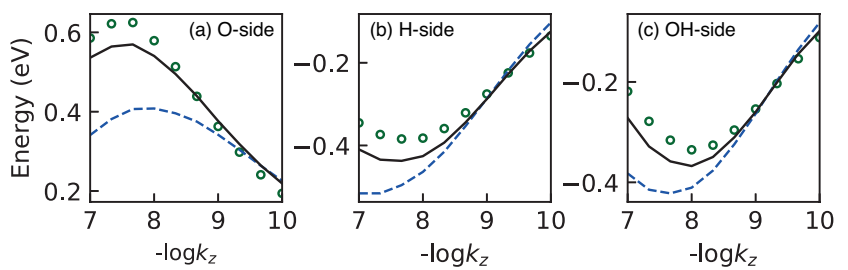

FIG. 1. Single water-electron interaction energies (Eq. 2) as a function of confinement strength, $k_{z}$ for three different orientations: (a) oxygen side, (b) dipole aligned, (c) $\mathrm{OH}$ aligned. Interaction energies from BLYP and BH\&HLYP (dashed blue and solid black curves respectively) are compared to MP2 results (green circles).

deed see that for all orientations and confining strengths, BLYP (dashed blue curves) underestimates the spin density of the excess electron in the attractive well region and overestimates the spin density on the water molecule compared to MP2 (green circles), with the error most severe at large confining strengths.

Johnson and co-workers found that using a hybrid functional with an increasing fraction of exact exchange reduced delocalization error. ${ }^{23,24}$ To test this, we plot interaction energies for the half-and-half hybrid, BH\&HLYP, ${ }^{32}$ as solid black curves in Fig. 1. We see much improved agreement to MP2 with this functional, with errors in the interaction energy not exceeding 0.06 $\mathrm{eV}$, although a small amount of delocalization error remains, as seen in the solid black curves of Fig. 2, and noted also by Johnson and co-workers. ${ }^{23,24}$ However, the good agreement between BH\&HLYP and MP2 interaction energies in Fig. 1, in addition to Herbert and HeadGordon's previous observation that this functional well reproduces vertical detachment energies of water cluster anions, ${ }^{33,34}$ suggests that the remaining delocalization error is not too severe, and this functional is useful in describing $e_{(\mathrm{aq})}^{-}$.

\section{COMPUTATIONAL DETAILS}

We built a spherical nanodroplet model of the condensed-phase $e_{(\mathrm{aq})}^{-}$following a similar protocol to the companion paper. ${ }^{21} \mathrm{~A}$ cavity for the excess electron was carved out by placing a chloride ion at the origin of a $25-\AA$ radius droplet of 2017 water molecules created using the SolvateCap command of tleap in the Amber 2018 package. ${ }^{35}$ The water forcefield was described by the SPC/Fw model, ${ }^{36}$ and chloride Lennard-Jones parameters were taken from Ref. 37. A half-harmonic cap potential with a force constant of $10 \mathrm{kcal} / \mathrm{mol} / \AA^{2}$ pre-

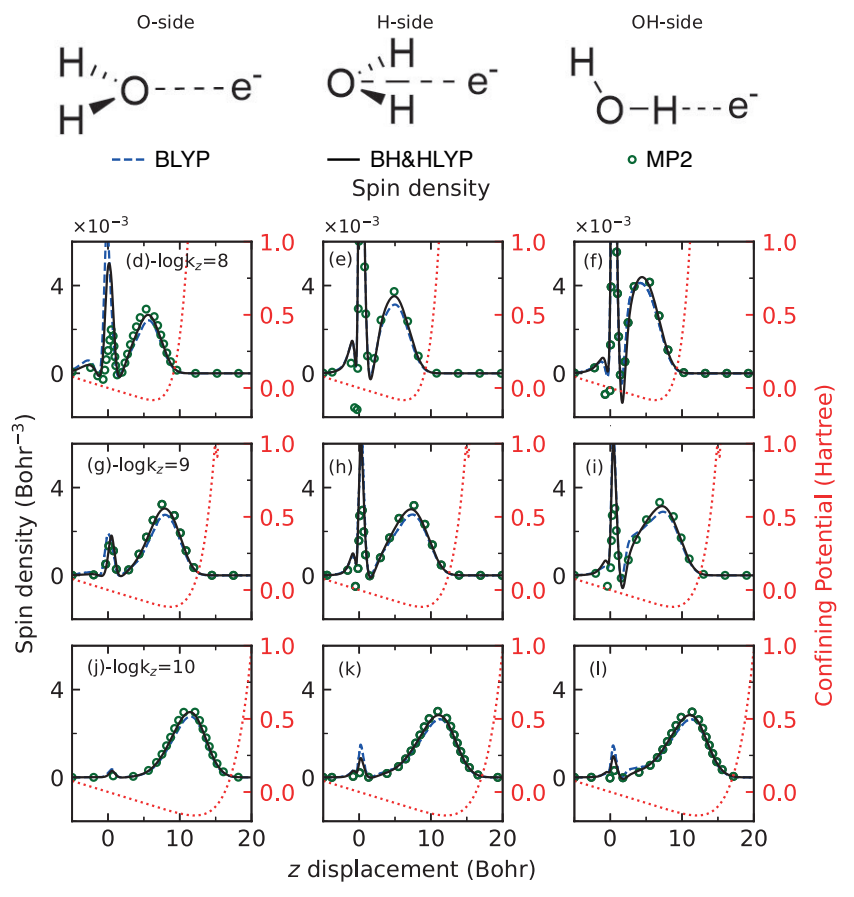

FIG. 2. Spin densities for a single water-electron system in the confining potential of Eq. 1. Three different orientations are considered, corresponding to the three columns and indicated by the molecular graphic at the top. Three values of confinement in the $z$ direction (electron-water direction) are shown as the three rows. BLYP and BH\&HLYP spin densities (dashed blue and solid black curves respectively) are compared to MP2 results (green circles). Also shown are the confining potentials (red dotted curve, right axis labels).

vented evaporation of water at the droplet/vacuum interface, and the chloride ion was restrained to the origin using a harmonic potential with a force constant of 1000 $\mathrm{kcal} / \mathrm{mol} / \AA^{2}$. Following minimization, heating, and equilibration, a 100-ps NVT production run was performed to generate ten independent snapshots separated by 10 ps, from which the chloride ion was then removed to generate initial conditions for the $e_{(\mathrm{aq})}^{-}$simulations.

To model $e_{(\mathrm{aq})}^{-}, 32$ water molecules nearest the origin of the droplet were treated at a QM level, with the remaining 1985 water molecules treated at an MM level. Since the switch from MM to QM introduced large forces on the QM water molecules, we quenched the largest components of their forces by performing 5 steps of minimization using the L-BFGS algorithm, ${ }^{38}$ implemented in DL-FIND. ${ }^{39}$ During dynamics, QM/MM separation was maintained with FlexiBLE implemented in a development version of Terachem, ${ }^{40-42}$ and interfaced with OpenMM 7.3. ${ }^{43}$ We used a FlexiBLE exponent parameter of $\alpha=15 \AA^{-1}$ and a convergence parameter of $\gamma=10^{-3} k_{\mathrm{B}} T$. The $\mathrm{QM}$ region was described at the BH\&HLYP-D3/6-31++ $\mathrm{G}^{*}$ level of theory, where D3 represents dispersion corrections with Becke-Johnson damping. ${ }^{44}$ AIMD was propagated with the velocity Verlet algorithm ${ }^{45}$ using the Niklasson time-reversible 
Born-Oppenheimer integrator with a 0.5 -fs timestep. ${ }^{46}$ Temperature was maintained at $298 \mathrm{~K}$ with the BussiParrinello thermostat. ${ }^{47}$ We generated ten trajectories of $7 \mathrm{ps}$ in length. The first 2 ps of data of each trajectory was discarded as an equilibration period.

We note that one challenge arising in the simulation of $e_{(\mathrm{aq})}^{-}$is the occasional rapid diffusion of the electron to the QM/MM boundary. This issue was noted previously by Holden et al, ${ }^{15}$ and we followed their solution of discarding trajectories that had the electron diffuse to the boundary, based on monitoring the outermost extent of the electron, defined as the sum of the electron's centroid distance:

$$
r_{0}(t)=\left|\left\langle\psi^{\mathrm{SOMO}}(t)|\hat{\boldsymbol{r}}| \psi^{\mathrm{SOMO}}(t)\right\rangle\right|
$$

and its radius of gyration:

$$
r_{\mathrm{gyr}}(t)=\left\langle\psi^{\mathrm{SOMO}}(t)\left|\left(\hat{\boldsymbol{r}}-\boldsymbol{r}_{0}(t)\right)^{2}\right| \psi^{\mathrm{SOMO}}(t)\right\rangle^{\frac{1}{2}},
$$

where $\psi^{\text {SOMO }}$ is the singly-occupied molecular orbital, occupied by the excess electron. If the sum of these values exceeded $6.0 \AA$, corresponding to the QM/MM boundary region, the trajectory was discarded. Fig. 3 shows an example of an accepted trajectory (black curve), in which the radial extent of the electron avoids the QM/MM interface. An example of a rejected trajectory (red curve) is also shown, in which the radial extent exceeded $6 \AA$ at $\sim 200$ fs. Discarding trajectories this way limits the length of our simulations to no more than several ps; however, this is sufficiently long to study the equilibrium dynamics of $e_{(\mathrm{aq})}^{-}$, which is dominated by solvent motions on sub-picosecond timescales.

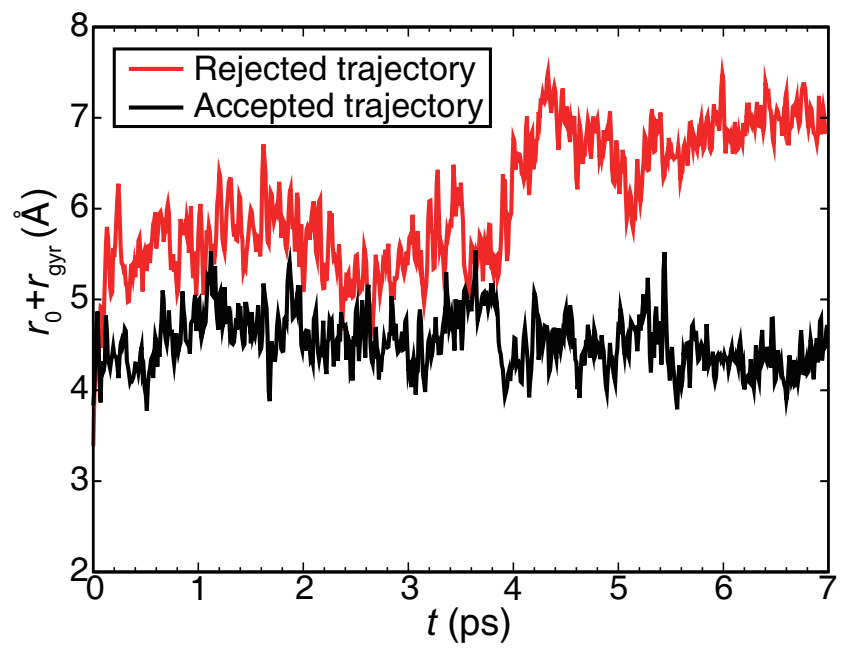

FIG. 3. Radial extent of the excess electron (sum of electron centroid, $r_{0}$, and radius of gyration $r_{\text {gyr }}$ ) shown for an accepted trajectory and a rejected trajectory (black and red curves respectively).

\section{RESULTS AND DISCUSSION}

\section{A. Liquid water structure}

Before considering $e_{(\mathrm{aq})}^{-}$, we first verified our simulation setup by performing AIMD on pure liquid water at the BH\&HLYP-D3/6-31+G* level. To our knowledge, the structure of water at the BH\&HLYP level has not been previously published. Using the same protocol discussed above, but without a chloride ion, we generated a spherical nanodroplet of 2028 waters. We fixed the oxygen atom of one QM water molecule at the origin, and allowed the other 31 QM and 1996 MM water molecules to move freely. FlexiBLE was used to maintain QM/MM separation, and 110-ps of NVT dynamics was propagated.

The Radial Distribution Functions (RDF) for liquid water relative to the central QM oxygen atom are shown in Fig. 4. Compared to the experimental data of Ref. 48, we see that the overall solvent structure of liquid water is well reproduced, albeit with some noticeable overstructuring in the first solvent shell compared to experiment. It is well known that such overstructuring arises from the neglect of Nuclear Quantum Effects (NQE), ${ }^{49-51}$ and thus we view the RDFs in Fig. 4 as indicating that $\mathrm{BH} \& \mathrm{HLYP} / 6-31+\mathrm{G}^{*}$ provides a faithful representation of liquid water's potential energy surface. Furthermore, we see that FlexiBLE has correctly maintained QM/MM separation (indicated by solid red and blue filled areas), without introducing artefacts in the total RDF.

\section{B. Hydrated electron structure}

Having verified that our simulation protocol gives a good description of liquid water's structure, we turn next to the simulated properties of $e_{(\mathrm{aq})}^{-}$. Fig. 5 shows the RDF for $e_{(\mathrm{aq})}^{-}$based on $e^{-}$-oxygen and $e^{-}$-hydrogen distances (panels (a) and (b) respectively). Encouragingly, $\mathrm{QM} / \mathrm{MM}$ separation is maintained as shown by the red and blue filled regions of Fig. 5. Comparing to Fig. 4, we note that there appears to be more overlap in the distributions of $\mathrm{QM} e^{-}-\mathrm{O}$ and $\mathrm{MM} e^{-}-\mathrm{O}$ distances compared to the respective $\mathrm{QM}$ and $\mathrm{MM}$ distributions of $\mathrm{O}-\mathrm{O}$ distances relative to a central fixed oxygen atom in the pure water simulations. This arises from diffusion of the excess electron's centroid position away from the center of the QM region (discussed above in Section III), rather than a breakdown of QM/MM separation.

In Fig. 5 we see the presence of a clear cavity in which the electron resides, with an $e^{-}-\mathrm{O}$ turn-on distance of $1.7 \AA$, and two strong peaks in the $e^{-}-\mathrm{O}$ RDF at 2.5 $\AA$ and $4.3 \AA$, indicating the first and second solvation shells. The $e^{-}-\mathrm{H}$ RDF displays a single strong peak at $1.5 \AA$, consistent with a predominantly $\mathrm{O}-\mathrm{H}$ bond aligned solvation of the excess electron. Integrating to the first minimum of the $e^{-}-\mathrm{O} \operatorname{RDF}$ at $3.3 \AA$ provides a coordination number of 4.0 , which can be compared to the 

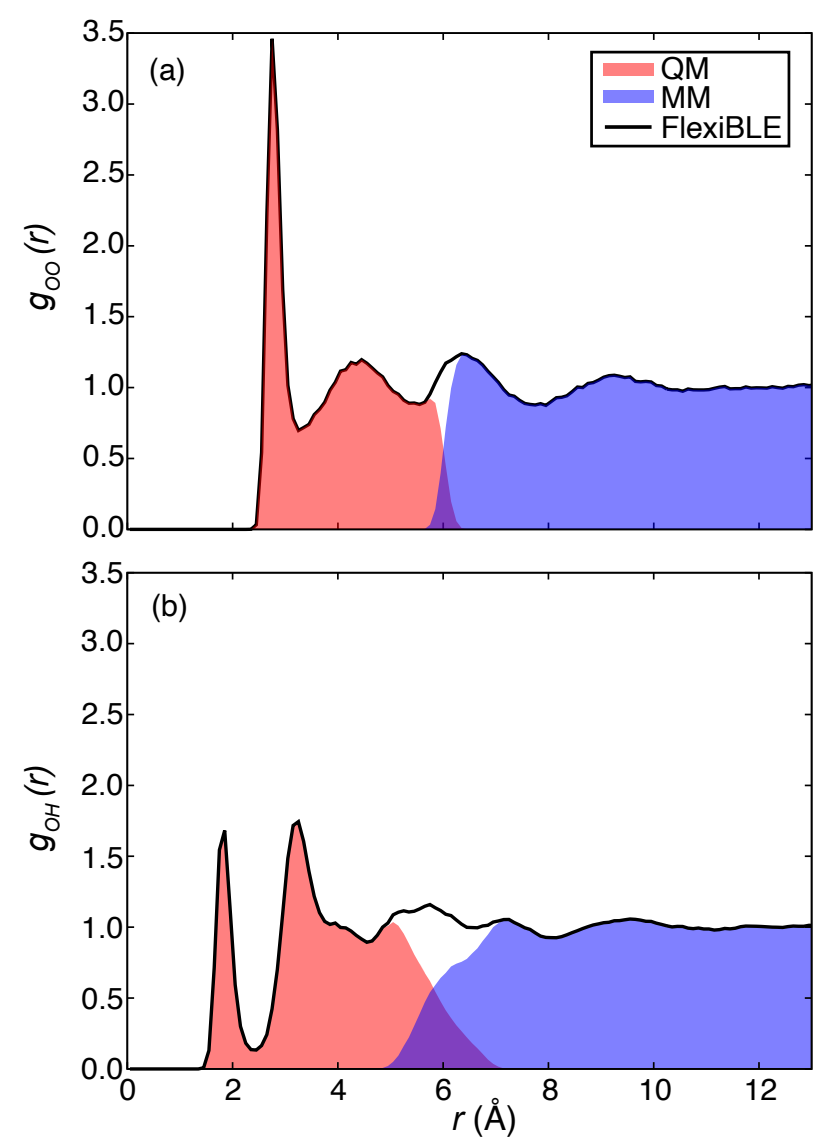

FIG. 4. RDF of liquid water at the FlexiBLE-QM/MM BH\&HLYP-D3/6-31+G* level. (a) Oxygen-Oxygen distribution (b) Oxygen-Hydrogen distribution. The breakdown of total RDFs (solid black curves) into QM and MM regions are shown by the shaded areas (red and blue respectively).

same procedure for the first minimum of the $e^{-} \mathrm{H} \mathrm{RDF}$ at $2.4 \AA$ which yields a coordination number of 3.9. The structure of $e_{(\mathrm{aq})}^{-}$is thus essentially the same as found in previous $\mathrm{QM} / \mathrm{MM}$ simulations at the self-interaction corrected BLYP-D level, ${ }^{14}$ and consistent with the overall picture provided by cavity-forming MQC simulations, ${ }^{4,7}$ and recent PIMD simulations with ML potentials, ${ }^{19}$ although the latter's prediction of quasi-stable dual cavity structures is not observed here, perhaps due to the neglect of NQE.

\section{Hydrated electron dynamics}

We turn next to the equilibrium dynamics of $e_{(\mathrm{aq})}^{-}$, which we measure through the time autocorrelation function of its band gap fluctuations:

$$
C_{E E}(t)=\frac{\langle\delta \Delta E(0) \delta \Delta E(t)\rangle}{\left\langle\delta \Delta E^{2}(0)\right\rangle},
$$
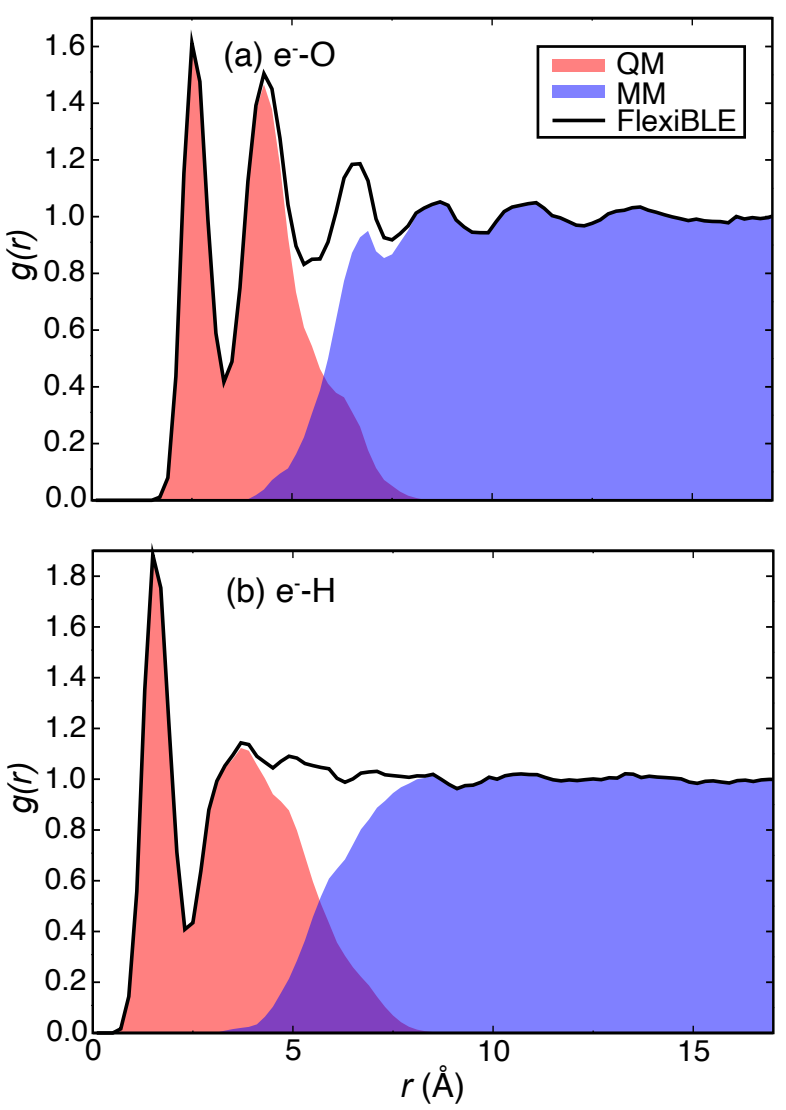

FIG. 5. AIMD hydrated electron RDF, divided into two regions (red-QM, blue-MM). (a) $e_{\mathrm{aq}}^{-}$-Oxygen (b) $e_{\mathrm{aq}}^{-}$-Hydrogen. Good QM/MM separation is maintained during the simulation. Strong solvent shell structure is observed for hydrated electron.

where the angled brackets indicate a classical ensemble average and the fluctuations in the band gap are defined as:

$$
\delta \Delta E(t)=E_{1}(t)-E_{0}(t)-\left\langle E_{1}-E_{0}\right\rangle,
$$

and $E_{i}$ is the energy of state $i$. For our DFT simulations, we take the band gap to be the HOMO-LUMO gap. For the MQC simulations, the band gap is the excitation energy between the lowest two eigenvalues of the one-electron Hamiltonian.

As discussed in paper I, $C_{E E}$ reports couplings between solvent motions and the energy levels of the excess electron, and FlexiBLE was seen to reproduce $C_{E E}$ from full-system calculations at the MQC level, indicating the bias potential did not measurably influence solvent motions that couple to the excess electron. ${ }^{21}$ To directly reveal the electron-coupled solvent motions, we take the Fourier transform of $C_{E E}(t)$, and display the result in Fig. 6(a). We focus on the frequency range of 1000-4000 $\mathrm{cm}^{-1}$, which reports couplings to the intramolecular vibrations of water, and allows comparison to experimental Resonance Raman (RR) spectroscopy. ${ }^{52}$ 


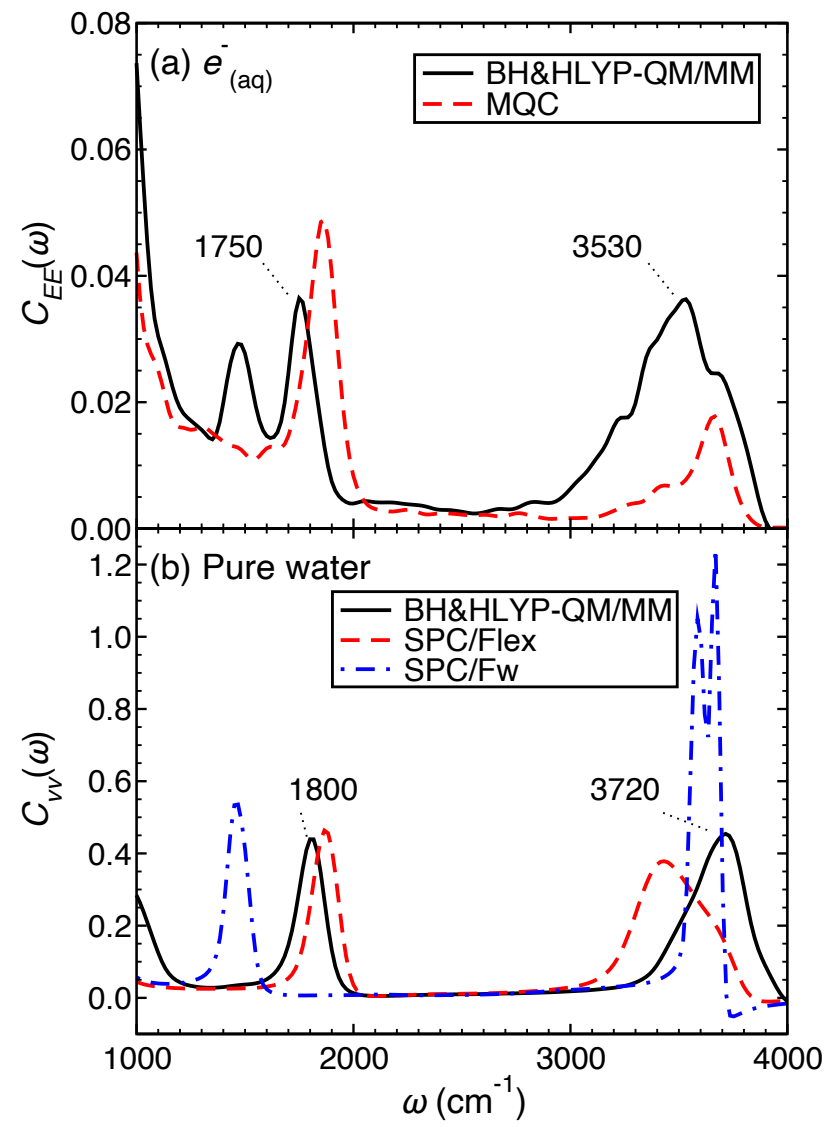

FIG. 6. Fourier transforms of (a) $e_{(\text {aq })}^{-}$'s band gap autocorrelation function, and (b) pure water's velocity autocorrelation function.

As Fig. 6(a) shows, the energy gap fluctuations of $e_{(\mathrm{aq})}^{-}$ at the FlexiBLE QM/MM level (solid black curve) are dominated by two features: a broad peak centered at $3530 \mathrm{~cm}^{-1}$, and a pair of peaks at 1500 and $1750 \mathrm{~cm}^{-1}$. The $3530 \mathrm{~cm}^{-1}$ peak is assigned to water's $\mathrm{OH}$ stretch, while the $1750 \mathrm{~cm}^{-1}$ peak corresponds to water's bend. These numbers are somewhat higher than the experimental $e_{(\mathrm{aq})}^{-} \mathrm{RR}$ peak frequencies of 1610 and $\sim 3200$ $\mathrm{cm}^{-1} ;^{52}$ however, applying the recommended vibrational frequency scaling factor of 0.9374 for $\mathrm{BH} \& \mathrm{HLYP}^{53}$ brings the theoretical frequencies into better agreement with experiment. We will address the $1500 \mathrm{~cm}^{-1}$ peak further below.

Comparing QM/MM to MQC results (solid black and dashed red curves of Fig. 6(a) respectively) we see the QM/MM peaks are red shifted and broadened compared to MQC. The MQC peaks align with the frequencies of the underlying SPC/Flex water model ${ }^{54}$ (dashed red curve in panel (b) which shows the Fourier transform of pure water's velocity autocorrelation function); in particular, agreement is seen with the bend and asymmetric stretch at 1865 and $3665 \mathrm{~cm}^{-1}$ respectively. On the other hand, the $e_{(\mathrm{aq})}^{-} \mathrm{QM} / \mathrm{MM}$ peaks are red shifted compared to the corresponding pure water frequencies at the FlexiBLE BH\&HLYP/6-31+G* QM/MM level (solid curve in panel (b)). The magnitudes of these excess electroninduced red shifts match very well with those seen in the experimental RR of $e_{(\mathrm{aq})}^{-}:{ }^{52} 50(\sim 30) \mathrm{cm}^{-1}$ for the bend and $190(200) \mathrm{cm}^{-1}$ for the stretch, with experimental numbers in brackets. The RR vibrational red shifts have been previously assigned to occupation of water's $\mathrm{OH}$ antibonding orbitals $\left(\sigma_{\mathrm{OH}}^{*}\right)$ by the excess electron, ${ }^{55}$ which we confirm below.

The spectral density of pure water with the SPC/Fw forcefield, shown as the dot dashed blue curve in Fig. 6(b), reveals the origin of the peak at $1500 \mathrm{~cm}^{-1}$ seen in the gap correlation function of $e_{(\mathrm{aq})}^{-}$at the FlexiBLE QM/MM level (solid black curve in Fig. 6(a)): this peak arises from coupling between the excess electron and the MM SPC/Fw water molecules' bend. SPC/Fw was parameterized to reproduce structural, thermodynamic, and kinetic, rather than vibrational, properties of liquid water, ${ }^{56}$ and the bend frequency is evidently underestimated in this model. Although our use of the $\mathrm{SPC} / \mathrm{Fw}$ model results in a spurious secondary water bend peak in $e_{(\mathrm{aq})}^{-}$'s energy gap fluctuations, we view this as serendipitous, as it allows us to easily separate the coupling of the excess electron to $\mathrm{QM}$ and $\mathrm{MM}$ water bend vibrations, which we see to be dominated by the former.

In addition to the redshifts between QM/MM and MQC results, another difference is that the total amplitude of coupling to the $\mathrm{OH}$ stretch at the $\mathrm{QM} / \mathrm{MM}$ level is significantly increased compared to MQC. Integrating the modulus squared of the Fourier transform between 2500 and $4000 \mathrm{~cm}^{-1}$ reveals that the coupling to the $\mathrm{OH}$ stretch is 8.6 times stronger in the QM/MM model compared to MQC. On the other hand, the coupling to the bend is comparable: between 1616 and $2500 \mathrm{~cm}^{-1}$, the MQC integral is 1.9 times larger than QM/MM. The ratio of MQC:QM/MM bend intensity reduces to 1.1 if the second QM/MM peak is included by extending the lower integration limit to $1350 \mathrm{~cm}^{-1}$.

The observation of electron induced vibrational redshifts and a significant enhancement of coupling to water's $\mathrm{OH}$ stretch compared to MQC results suggests that the excess electron interacts with water much more intimately than simple electrostatics would suggest. The vibrational redshifts arise from occupation of $\sigma_{\mathrm{OH}}^{*}$ orbitals, ${ }^{55}$ the energy of which is strongly modulated by the $\mathrm{OH}$ stretch mode. It is thus reasonable to expect that $\sigma_{\mathrm{OH}}^{*}$ occupation also explains the enhanced coupling between the electron and stretch modes. To explore this further, in the next section we seek to quantify the occupation of $\sigma_{\mathrm{OH}}^{*}$ orbitals, using $\mathrm{NBO}$ analysis.

\section{NBO analysis}

We performed NBO analysis at the BH\&HLYP/6$311++\mathrm{G}^{* *}$ level on $e_{(\mathrm{aq})}^{-}$snapshots separated by $100 \mathrm{fs}$ 
extracted from our BH\&HLYP QM/MM trajectories using NBO 7.0.8, ${ }^{57}$ interfaced with Q-Chem 5.0.2. ${ }^{28}$ Special care is needed in the NBO analysis of solvent-supported states, such as $e_{(\mathrm{aq})}^{-}$, since the default approach is to optimize spin NBOs to each have approximately unit occupation. For $e_{(\mathrm{aq})}^{-}$, this resulted in a single water close to the electron having a Lewis structure of $\mathrm{H}^{*} \mathrm{OH}^{-}$; i.e., with a broken $\mathrm{OH}$ bond, and with the excess charge assigned to the resulting hydroxide ion. Since we do not observe $\mathrm{OH}$ bond breaking in our AIMD simulations, we view this Lewis structure as unphysical. This behavior was avoided by specifying in the input a Lewis structure that leaves water molecules intact.

A second issue in the $\mathrm{NBO}$ analysis of $e_{(\mathrm{aq})}^{-}$relates to the diffuse nature of the excess electron's orbital and its occupation of a solvent cavity. Using an exclusively atom-centered basis augmented with diffuse functions $\left(6-311++\mathrm{G}^{* *}\right)$, we found significant mixing in of Rydberg-like character in the $\sigma_{\mathrm{OH}}^{*} \mathrm{NBOs}$ of the water molecules closest to the electron (see Supplementary Material Fig. S1), resulting in artificially high $\sigma_{\mathrm{OH}}^{*}$ occupations of $>0.3$. We found this issue was circumvented by adding a ghost atom with lithium's $6-311++\mathrm{G}^{* *}$ basis functions (chosen as the element with the smallest diffuse exponent) at the centroid position of the SOMO. We then assigned the excess electron to an NBO formed largely from the lithium ghost atom's outer valence and diffuse set, with smaller contributions from Rydberg natural atomic orbitals of the water atoms. We identify this ghost atom NBO as an $s$-like cavity orbital, $\mathrm{Cav}_{s}$. Representative $\sigma_{\mathrm{OH}}^{*}$ and $\mathrm{Cav}_{s}$ NBOs are shown in Fig. 7 . Convergence of NBO properties with basis set and QM region size is explored in Section S-I, where we show that our chosen $6-311++\mathrm{G}^{* *}$ basis provides comparable results to aug-cc-pVQZ, but at significantly lower computational cost, allowing the full QM region of 32 water molecules to be included.

We start by considering average NBO populations of the $\mathrm{Cav}_{s}$ and $\sigma_{\mathrm{OH}}^{*}$ orbitals after ordering $\alpha$-spin NBOs by population for each snapshot, with the highest populated antibonding orbital labelled as $\sigma_{1}^{*}$. The result is shown in Table I. As expected, the $\mathrm{Cav}_{s}$ orbital has the highest population; however, $\sigma_{\mathrm{OH}}^{*}$ orbitals contribute an appreciable $\sim 30 \%$. In fact, the $\mathrm{Cav}_{s}$ and five $\sigma_{\mathrm{OH}}^{*}$ orbitals sum to a population of 0.97 , and thus together describe the excess electron's SOMO orbital almost in its entirety. The remaining amplitude of the SOMO is made up of contributions from lower-populated $\sigma_{\mathrm{OH}}^{*}$ orbitals and water Rydberg orbitals. This analysis qualitatively supports the picture of $e_{(\mathrm{aq})}^{-}$put forward by Uhlig, Marsalek, and Jungwirth (UMJ), who, based on a partitioning of spin density, assigned $41 \%$ of the electron to the cavity region, $24 \%$ assigned to a region overlapping with the first two solvation shells water molecules, and $35 \%$ assigned to a diffuse tail extending beyond the second solvation shell. ${ }^{14} \mathrm{We}$ assign a larger contribution to a cavity state than UMJ, since the $\mathrm{Cav}_{s} \mathrm{NBO}$ also con-

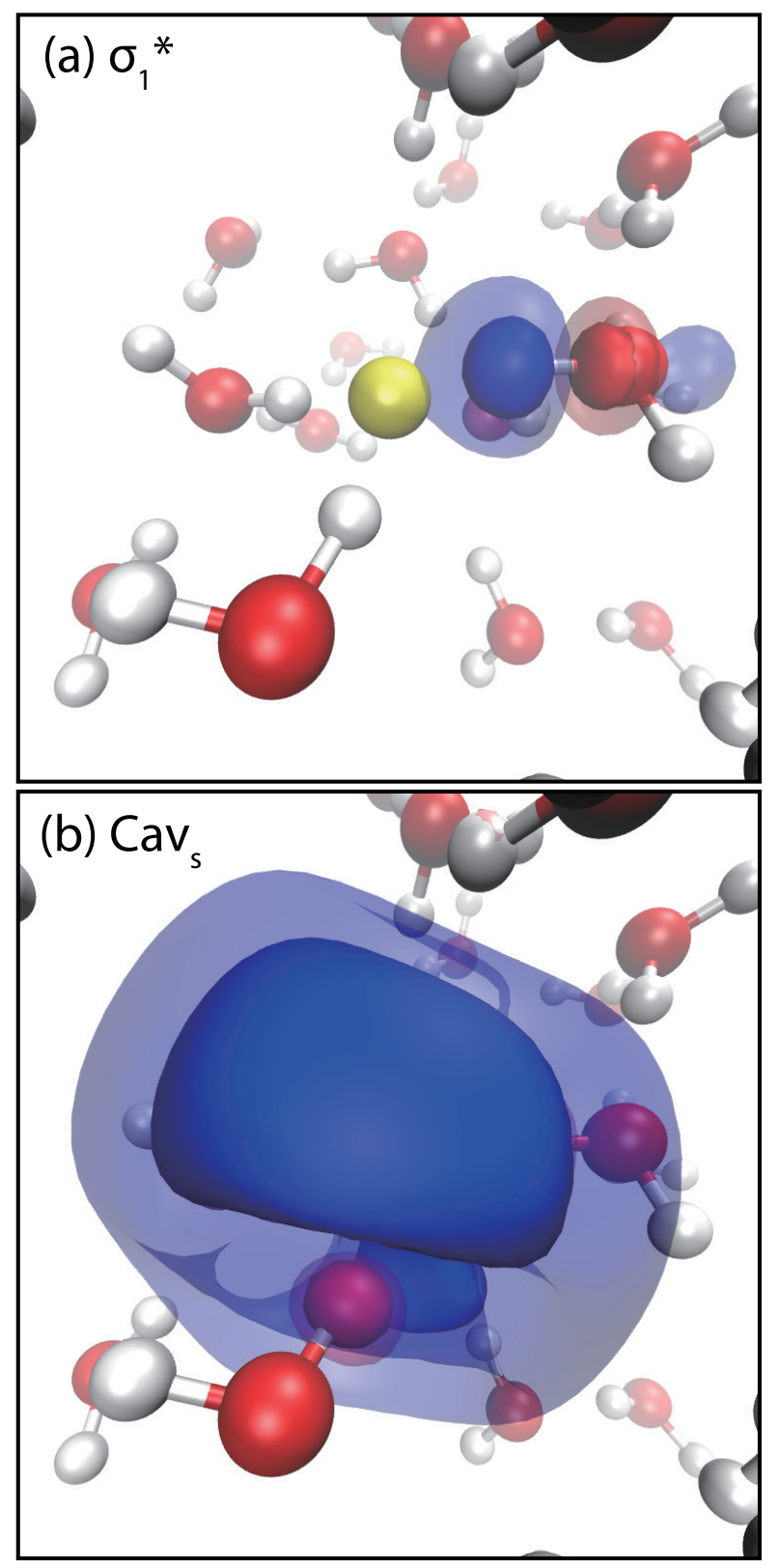

FIG. 7. Visualization of $e_{(\mathrm{aq})}^{-}$'s (a) $\sigma_{\mathrm{OH}}^{*}$ and (b) $s$-like cavity orbitals from NBO analysis. The transparent and solid surfaces enclose $75 \%$ and $50 \%$ respectively of a single electron's density. The yellow sphere indicates the centroid position of the SOMO.

tains the diffuse tails that UMJ assigned separately. Our analysis provides additional quantitative insight into how the excess electron is able to have appreciable amplitude in the first two solvation shells, which is by populating the $\sigma_{\mathrm{OH}}^{*}$ orbitals.

To further explore the magnitude of $\sigma_{\mathrm{OH}}^{*}$ occupation in $e_{(\mathrm{aq})}^{-}$, we found it revealing to project the distribution of occupations onto the $e^{-}$-oxygen distance and $e^{-}-\mathrm{O}-\mathrm{H}$ angle, where the excess electron's position is taken to be the centroid of the SOMO. This is shown as the false 


\begin{tabular}{|c|c|}
\hline$\overline{\mathrm{NBO}}$ & opulation(Stdev) \\
\hline $\mathrm{Cav}_{\mathrm{s}}$ & $0.675(0.055)$ \\
\hline$\sigma_{1}^{*}$ & $0.106(0.029)$ \\
\hline$\sigma_{2}^{*}$ & $0.074(0.019)$ \\
\hline$\sigma_{3}^{*}$ & $0.053(0.016)$ \\
\hline$\sigma_{4}^{*}$ & $0.036(0.012)$ \\
\hline$\sigma_{5}^{*}$ & $0.026(0.005)$ \\
\hline
\end{tabular}

TABLE I. Average populations of $\alpha$-spin NBOs in $e_{(\mathrm{aq})}^{-}$. Numbers in brackets represent standard deviations.

color map in Fig. 8. Surprisingly, we see $\sigma_{\mathrm{OH}}^{*}$ occupations on a single $\mathrm{OH}$ bond reach 0.2 . This high occupation occurs exclusively when a water molecule is close to the electron $\left(e^{-}\right.$-O distance $\left.<2 \AA\right)$ and with the $\mathrm{OH}$ bond aligned to the electron $\left(e^{-}-\mathrm{O}-\mathrm{H}\right.$ angle $\left.<20^{\circ}\right)$. The remaining first-solvent shell water molecules $\left(e^{-}-\mathrm{O}\right.$ distance $<3 \AA$ ) exhibit moderately large $\sigma_{\mathrm{OH}}^{*}$ occupations of $\sim 0.1$, but again only for the $\mathrm{OH}$ bond aligned to the electron. The other $\mathrm{OH}$ bonds of the first-shell waters do not have appreciable $\sigma_{\mathrm{OH}}^{*}$ occupation, nor do second shell or further water molecules.

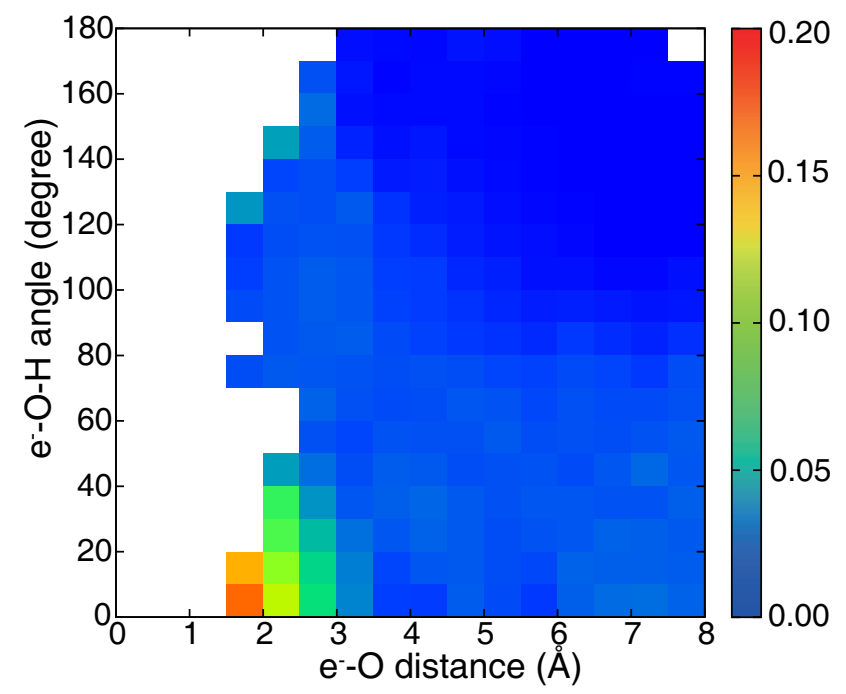

FIG. 8. Distribution of $\sigma_{\mathrm{OH}}^{*}$ occupation per $\mathrm{OH}$ bond projected onto $e^{-}$-oxygen distance and $e^{-}-\mathrm{O}-\mathrm{H}$ angle. White colored regions indicate the absence of a water molecule at that distance/angle.

Although a relatively rare event (see Fig. S2), $\sigma_{\mathrm{OH}}^{*}$ occupations of 0.2 are surprisingly high, and exceed values found even in $\mathrm{H}_{2} \mathrm{O}-\mathrm{OH}^{-}$complexes (0.17) and water cluster anion isomers exhibiting the double acceptor (AA) motif (0.16). ${ }^{22}$ The latter are associated with water stretch vibrational redshifts of $>300 \mathrm{~cm}^{-1} .58$ It stands to reason then that similarly large, or larger, vibrational redshifts occur in the condensed-phase $e_{(\mathrm{aq})}^{-}$. The overall $200 \mathrm{~cm}^{-1}$ redshift we observe in the $\mathrm{OH}$ stretch of $e_{(\mathrm{aq})}^{-}$thus appears to result from the smaller av- erage $\sigma_{\mathrm{OH}}^{*}$ populations of $\sim 0.05-0.1$ seen in the first-shell water molecules. However, the spectral envelope of the $\mathrm{OH}$ stretch is seen to extend down to $\sim 3000 \mathrm{~cm}^{-1}$ (see Fig. 6(a)) and we hypothesize that these strongly redshifted frequencies are due to the water molecule closest to the electron when it has a high $\sigma_{\mathrm{OH}}^{*}$ occupation. This idea will be explored in more detail in a future publication.

The observed large $\sigma_{\mathrm{OH}}^{*}$ occupations of 0.20 are further surprising considering the energy levels involved. The hydrated electron state has an energy of $-3.7 \mathrm{eV}$ relative to vacuum, ${ }^{59,60}$ while the $\sigma_{\mathrm{OH}}^{*}$ orbitals are several $\mathrm{eV}$ above the vacuum level. ${ }^{61}$ For the hydrated electron state to have significant $\sigma_{\mathrm{OH}}^{*}$ occupation then suggests a very large Hamiltonian coupling (several eV) of the excess electron to the $\sigma_{\mathrm{OH}}^{*}$ orbitals. NBO analysis allows for a determination of Hamiltonian couplings by computing matrix elements of the Fock operator in the NBO basis. We averaged these matrix elements over our FlexiBLE QM/MM trajectories. ${ }^{62}$ The results are presented in Table II which shows matrix elements between the $\mathrm{Cav}_{\mathrm{s}}$ orbital and the five most populated $\sigma_{\mathrm{OH}}^{*}$ orbitals. For simplicity, matrix elements coupling the $\sigma_{\mathrm{OH}}^{*}$ orbitals with each other are omitted.

\begin{tabular}{|c|c|c|c|c|c|c|}
\hline & $\mathbf{C a v}_{\mathbf{s}}$ & $\boldsymbol{\sigma}_{\mathbf{1}}^{*}$ & $\boldsymbol{\sigma}_{\mathbf{2}}^{*}$ & $\boldsymbol{\sigma}_{\mathbf{3}}^{*}$ & $\boldsymbol{\sigma}_{\mathbf{4}}^{*}$ & $\boldsymbol{\sigma}_{\mathbf{5}}^{*}$ \\
\hline $\mathbf{C a v}_{\mathbf{s}}$ & 2.63 & 4.49 & 4.03 & 3.52 & 2.50 & 0.33 \\
\hline $\boldsymbol{\sigma}_{\mathbf{1}}^{*}$ & 4.49 & 13.27 & 0.00 & 0.00 & 0.00 & 0.00 \\
\hline $\boldsymbol{\sigma}_{\mathbf{2}}^{*}$ & 4.03 & 0.00 & 13.94 & 0.00 & 0.00 & 0.00 \\
\hline $\boldsymbol{\sigma}_{\mathbf{3}}^{*}$ & 3.52 & 0.00 & 0.00 & 14.22 & 0.00 & 0.00 \\
\hline $\boldsymbol{\sigma}_{\mathbf{4}}^{*}$ & 2.50 & 0.00 & 0.00 & 0.00 & 14.21 & 0.00 \\
\hline $\boldsymbol{\sigma}_{\mathbf{5}}^{*}$ & 0.33 & 0.00 & 0.00 & 0.00 & 0.00 & 14.18 \\
\hline
\end{tabular}

TABLE II. Model Hamiltonian of $e_{(\mathrm{aq})}^{-}$in a basis of an $s$-like cavity orbital and the five $\sigma^{*}$ orbitals with largest coupling to the cavity orbital. All values are in $\mathrm{eV}$.

We consider first the diagonal elements of the model Hamiltonian in Table II, where we observe that all orbitals are above the vacuum level. This makes physical sense for the $\sigma_{\mathrm{OH}}^{*}$ orbitals, but might at first seem surprising for the cavity orbital. However, this cavity orbital is by construction orthogonal to all water-centered occupied and virtual NBOs and is thus rather confined in the cavity region and interstitial regions between the water molecules, raising its kinetic energy.

Considering the off-diagonal elements of the Hamiltonian in Table II, we indeed find that the $\mathrm{Cav}_{s}$ and $\sigma_{\mathrm{OH}}^{*}$ orbitals are coupled by up to several $\mathrm{eV}$, with the largest average coupling of $4.49 \mathrm{eV}$ found for $\sigma_{1}^{*}$, which is also the closest $\mathrm{OH}$ bond to the electron. Similarly large couplings are seen for $\sigma_{2}^{*}$ through $\sigma_{4}^{*}$, but by $\sigma_{5}^{*}$, the coupling has dropped to $0.33 \mathrm{eV}$. These values are consistent with the inner solvent shell of $e_{(\mathrm{aq})}^{-}$comprising four $\mathrm{OH}-$ aligned water molecules. The couplings may be viewed rather like Charge-Transfer (CT) integrals between the donor $\mathrm{Cav}_{s}$ orbital and acceptor $\sigma_{\mathrm{OH}}^{*}$ orbitals. In this 
CT context, the large magnitude of the couplings can be understood as resulting from two aspects of $e_{(\mathrm{aq})}^{-}$. First, the diffuse nature of the $\mathrm{Cav}_{s}$ orbital provides a high degree of spatial overlap with the acceptor $\sigma_{\mathrm{OH}}^{*}$ of the first solvent shell, which explains why the coupling drops off for the fifth and further $\sigma_{\mathrm{OH}}^{*}$ orbitals. Second, the transition density connecting the $\mathrm{Cav}_{s}$ and $\sigma_{\mathrm{OH}}^{*}$ orbitals is localized near the partially positive $\mathrm{H}$ atoms (see Fig S3), which results in an attractive Coulombic coupling.

Diagonalizing the Hamiltonian in Table II yields a lowest eigenvalue of $-1.08 \mathrm{eV}$, which while higher than the true average SOMO energy of $-2.56 \mathrm{eV}$, shows that our simple model captures most of the stabilization of the cavity orbital by strongly coupling to four $\sigma_{\mathrm{OH}}^{*}$ orbitals (see Fig. S4 for convergence of the eigenvalue with number of included $\sigma_{\mathrm{OH}}^{*}$ orbitals and Fig. S5 for its correlation with the SOMO energy). ${ }^{63}$

The energy levels of the NBOs and model Hamiltonian eigenvalues are summarized in the MO diagram presented in Fig. 9. We notice an interesting stabilization of the most populated $\sigma_{1}^{*}$ orbital's energy of $\sim 0.9 \mathrm{eV}$ relative to the other $\sigma_{\mathrm{OH}}^{*}$ orbitals, that all have average energies of $\sim 14.2 \mathrm{eV}$. We found this stabilization arises due to a lengthening of the closest $\mathrm{OH}$ bond to the electron, which had an average length of $0.999 \pm 0.002 \AA$ compared to the remaining QM OH bonds which had an average length of $0.9761 \pm 0.0003 \AA . \sigma_{2}^{*}$ is stabilized by $\sim 0.3 \mathrm{eV}$ in a similar manner, but to a lesser degree. Thus, the partial occupation of water $\sigma_{\mathrm{OH}}^{*}$ orbitals is seen to noticeably weaken the coordinating $\mathrm{OH}$ bonds, which lowers the $\sigma_{\mathrm{OH}}^{*}$ orbital energies and further enhances their coupling to the excess electron. This physics is missed entirely by MQC models, and provides an explanation for the significant differences in $e_{(\mathrm{aq})}^{-}$'s dynamics we observe at the MQC and QM/MM levels.

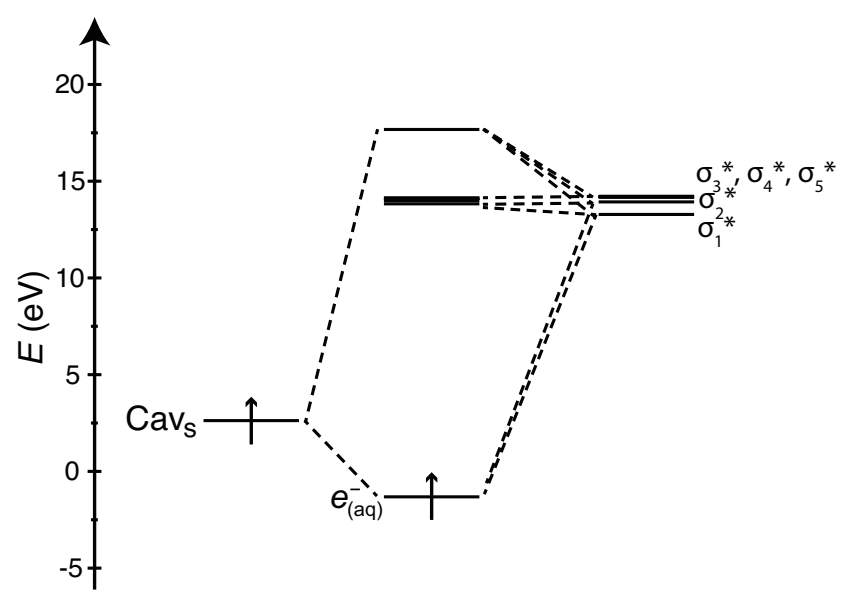

FIG. 9. MO diagram of $e_{(\mathrm{aq})}^{-}$from the model Hamiltonian in Table II.

\section{CONCLUSIONS}

In this paper, we applied the FlexiBLE embedding approach, developed in the preceding companion paper, ${ }^{21}$ to perform QM/MM dynamics of the hydrated electron at the BH\&HLYP/6-31++ $\mathrm{G}^{*}$ level. We found agreement with the structural picture of $e_{(\mathrm{aq})}^{-}$put forward by UMJ, ${ }^{14}$ that the excess electron occupies a cavity, but has a complex character involving significant overlap with first-shell coordinating water molecules. Using NBO analysis, we provided a quantitative rationale for this complex behavior: the excess electron has $\sim 30 \%$ occupation of $\sigma_{\mathrm{OH}}^{*}$ orbitals brought about by large electronic couplings of a cavity state and the anti-bonding orbitals. This leads to an enhanced dynamical coupling between the excess electron's energy levels and water stretch vibrations compared to a MQC model. Furthermore, the weakening of $\mathrm{OH}$ bonds by $\sigma_{\mathrm{OH}}^{*}$ occupation leads to stretch and bend vibrations that are redshifted compared to pure water, in quantitative agreement with experiment. ${ }^{52}$ Surprisingly, the $\sigma_{\mathrm{OH}}^{*}$ occupation of a single water molecule can reach $20 \%$, which leads to a noticeable extension of that $\mathrm{OH}$ bond.

To answer the question posed in the introduction, we conclude that $e_{(\mathrm{aq})}^{-}$is not a simple spectator species, but rather is intimately coupled to, and strongly modulates, the motions of the first solvation shell waters. Previous MQC dynamics studies, which inherently miss this physics, emphasized the role of water translations and librations in the solvation dynamics of $e_{(\mathrm{aq})}^{-}$. Our results motivate a deeper consideration of intramolecular water motions. Overall, we believe that this successful first application demonstrates that FlexiBLE opens the door to studying the dynamics of many other condensed-phase species, for which a QM description of the environment is necessary.

\section{SUPPLEMENTARY MATERIAL}

See the supplementary material for further details on our NBO analysis and model Hamiltonian.

\section{ACKNOWLEDGMENTS}

This work was supported by the National Natural Science Foundation of China Young Scientist Fund (Grant No. 21603145), the Science and Technology Commission of Shanghai Municipality Foreign Experts Program (Grant No. 21WZ2503600), the NYU-ECNU Center for Computational Chemistry, and start-up funds from NYU Shanghai. 


\section{DATA AVAILABILITY}

The data that support the findings of this study are available within the article and its supplementary material. Molecular coordinates are available from the corresponding author upon reasonable request.

\section{REFERENCES}

${ }^{1}$ P. J. Rossky and J. Schnitker, J. Phys. Chem. 92, 4277 (1988). ${ }^{2}$ B. J. Schwartz and P. J. Rossky, J. Chem. Phys. 101, 6902 (1994). ${ }^{3}$ B. J. Schwartz and P. J. Rossky, J. Chem. Phys. 101, 6917 (1994). ${ }^{4}$ L. Turi and D. Borgis, J. Chem. Phys. 117, 6186 (2002).

${ }^{5}$ D. Borgis, P. J. Rossky, and L. Turi, J. Chem. Phys. 127, 174508 (2007).

${ }^{6}$ L. D. Jacobson, C. F. Williams, and J. M. Herbert, J. Chem. Phys. 130, 124115 (2009).

${ }^{7}$ L. D. Jacobson and J. M. Herbert, J. Chem. Phys. 133, 154506 (2010).

${ }^{8}$ R. E. Larsen, W. J. Glover, and B. J. Schwartz, Science 329, 65 (2010).

${ }^{9}$ W. J. Glover and B. J. Schwartz, J. Chem. Theory Comput. 12, 5117 (2016).

${ }^{10}$ C.-C. Zho, E. P. Farr, W. J. Glover, and B. J. Schwartz, J. Chem. Phys. 147, 074503 (2017).

${ }^{11}$ L. D. Jacobson and J. M. Herbert, Science 331, 1387 (2011).

${ }^{12}$ R. E. Larsen, W. J. Glover, and B. J. Schwartz, Science 331, 1387 (2011).

${ }^{13}$ W. J. Glover and B. J. Schwartz, J. Chem. Theory Comput. 16, 1263 (2020).

${ }^{14}$ F. Uhlig, O. Marsalek, and P. Jungwirth, J. Phys. Chem. Lett. 3, 3071 (2012).

${ }^{15}$ Z. C. Holden, B. Rana, and J. M. Herbert, J. Chem. Phys. 150, 144115 (2019).

${ }^{16}$ M. Boero, M. Parrinello, K. Terakura, T. Ikeshoji, and C. C. Liew, Phys. Rev. Lett. 90, 226403 (2003).

${ }^{17}$ F. Ambrosio, G. Miceli, and A. Pasquarello, J. Phys. Chem. Lett. 8, 2055 (2017)

18 J. Wilhelm, J. VandeVondele, and V. V. Rybkin, Angew. Chem. Int. Ed. 58, 3890 (2019).

${ }^{19}$ J. Lan, V. Kapil, P. Gasparotto, M. Ceriotti, M. Iannuzzi, and V. V. Rybkin, Nat. Comm. 12, 766 (2021).

${ }^{20}$ J. Savolainen, F. Uhlig, S. Ahmed, P. Hamm, and P. Jungwirth, Nat. Chem. 6, 697 (2014)

${ }^{21}$ Z. Shen and W. J. Glover, J. Chem. Phys. X (2021).

${ }^{22}$ J. M. Herbert and M. Head-Gordon, J. Amer. Chem. Soc. 128, 13932 (2006)

${ }^{23}$ E. R. Johnson, A. Otero-de-la Roza, and S. G. Dale, J. Chem. Phys. 139, 184116 (2013).

${ }^{24}$ S. G. Dale and E. R. Johnson, J. Chem. Phys. 143, 184112 (2015).

${ }^{25}$ The linear term, $C_{z} \hat{z}$ in Eq. 1 does alter the electronic structure of water in the form of polarization; however, the magnitude of $C_{z}$ is comparable to the electric fields experienced in liquid water.

${ }^{26}$ R. A. Kendall, T. H. Dunning, and R. J. Harrison, J. Chem. Phys. 96, 6796 (1992).

${ }^{27}$ K. Raghavachari, G. W. Trucks, J. A. Pople, and M. HeadGordon, Chem. Phys. Lett. 157, 479 (1989).

${ }^{28}$ Y. Shao, Z. Gan, E. Epifanovsky, A. T. B. Gilbert, M. Wormit, J. Kussmann, A. W. Lange, A. Behn, J. Deng, and X. Feng, Mol. Phys. 113, 184 (2015).

${ }^{29}$ P. M. Gill, B. G. Johnson, and J. A. Pople, Chem. Phys. Lett. 209, 506 (1993).

${ }^{30}$ A. D. Becke, Phys. Rev. A 38, 3098 (1988).
${ }^{31}$ C. Lee, W. Yang, and R. G. Parr, Phys. Rev. B 37, 785 (1988).

${ }^{32}$ A. D. Becke, J. Chem. Phys. 98, 1372 (1993).

${ }^{33}$ J. M. Herbert and M. Head-Gordon, J. Phys. Chem. A 109, 5217 (2005), pMID: 16833879 .

${ }^{34}$ J. M. Herbert and M. Head-Gordon, Phys. Chem. Chem. Phys. 8, 68 (2006).

${ }^{35}$ D. Case, I. Ben-Shalom, S. R. Brozell, D. S. Cerutti, T. Cheatham, V. W. D. Cruzeiro, T. Darden, R. Duke, D. Ghoreishi, M. Gilson, H. Gohlke, A. Götz, D. Greene, R. Harris, N. Homeyer, Y. Huang, S. Izadi, A. Kovalenko, T. Kurtzman, and P. A. Kollman, Amber 2018 (University of California, San Francisco, 2018).

${ }^{36}$ Y. Wu, H. L. Tepper, and G. A. Voth, J. Chem. Phys. 124, 024503 (2006).

${ }^{37}$ I. S. Joung and T. E. Cheatham, J. Phys. Chem. B 112, 9020 (2008).

${ }^{38}$ J. Nocedal, Math. Comput. 35, 773 (1980).

${ }^{39}$ J. Kästner, J. M. Carr, T. W. Keal, W. Thiel, A. Wander, and P. Sherwood, J. Phys. Chem. A 113, 11856 (2009).

${ }^{40}$ I. S. Ufimtsev and T. J. Martínez, J. Chem. Theory Comput. 4, $222(2008)$.

${ }^{41}$ I. S. Ufimtsev and T. J. Martinez, J. Chem. Theory Comput. 5, 1004 (2009).

${ }^{42}$ I. S. Ufimtsev and T. J. Martinez, J. Chem. Theory Comput. 5, 2619 (2009)

${ }^{43}$ P. Eastman, J. Swails, J. D. Chodera, R. T. McGibbon, Y. Zhao, K. A. Beauchamp, L.-P. Wang, A. C. Simmonett, M. P. Harrigan, C. D. Stern, R. P. Wiewiora, B. R. Brooks, and V. S. Pande, PLOS Comput. Biol. 13, e1005659 (2017).

${ }^{44}$ S. Grimme, S. Ehrlich, and L. Goerigk, J. Comput. Chem. 32, 1456 (2011)

${ }^{45}$ W. C. Swope, H. C. Andersen, P. H. Berens, and K. R. Wilson, J. Chem. Phys. 76, 637 (1982).

${ }^{46}$ A. M. N. Niklasson, P. Steneteg, A. Odell, N. Bock, M. Challacombe, C. J. Tymczak, E. Holmström, G. Zheng, and V. Weber, J. Chem. Phys. 130, 214109 (2009).

${ }^{47}$ G. Bussi, D. Donadio, and M. Parrinello, J. Chem. Phys. 126, 014101 (2007).

${ }^{48}$ D. H. Brookes and T. Head-Gordon, J. Phys. Chem. Lett. 6, 2938 (2015).

49 J. Lobaugh and G. A. Voth, J. Chem. Phys. 106, 2400 (1997).

${ }^{50}$ T. F. Miller and D. E. Manolopoulos, J. Chem. Phys. 123, 154504 (2005).

${ }^{51}$ J. Liu, W. H. Miller, G. S. Fanourgakis, S. S. Xantheas, S. Imoto, and S. Saito, J. Chem. Phys. 135, 244503 (2011).

${ }^{52}$ M. J. Tauber and R. A. Mathies, J. Amer. Chem. Soc. 125, 1394 (2003).

${ }^{53}$ M. L. Laury, M. J. Carlson, and A. K. Wilson, J. Comp. Chem. 33, 2380 (2012).

${ }^{54}$ K. Toukan and A. Rahman, Phys. Rev. B 31, 2643 (1985).

${ }^{55}$ S. Dasgupta, B. Rana, and J. M. Herbert, J. Phys. Chem. B 123, 8074 (2019).

${ }^{56} \mathrm{Q}$. Wu and T. Van Voorhis, J. Chem. Theory Comput. 2, 765 (2006).

${ }^{57}$ E. D. Glendening, C. R. Landis, and F. Weinhold, J. Comput. Chem. 40, 2234 (2019).

${ }^{58}$ N. I. Hammer, J. R. Roscioli, J. C. Bopp, J. M. Headrick, and M. A. Johnson, J. Chem. Phys. 123, 244311 (2005).

${ }^{59}$ D. Luckhaus, Y.-i. Yamamoto, T. Suzuki, and R. Signorell, Sci. Adv. 3, e1603224 (2017).

${ }^{60}$ J. Nishitani, Y.-i. Yamamoto, C. W. West, S. Karashima, and T. Suzuki, Sci. Adv. 5, EAAW6896 (2019).

${ }^{61}$ T. Fransson, Y. Harada, N. Kosugi, N. A. Besley, B. Winter, J. J. Rehr, L. G. M. Pettersson, and A. Nilsson, Chem. Rev. 116, 7551 (2016)

${ }^{62} \mathrm{NBO}$ analysis applies a phase consistency to the orbitals so that the sign of the off-diagonals of our Hamiltonian was consistent from snapshot to snapshot.

${ }^{63}$ The remaining discrepancy between the model eigenvalue and true SOMO energy presumably results from neglecting couplings between the $\sigma_{\mathrm{OH}}^{*}$ orbitals. 